This item was submitted to Loughborough's Research Repository by the author.

Items in Figshare are protected by copyright, with all rights reserved, unless otherwise indicated.

\title{
Angle-resolved photoemission spectroscopy of band tails in lightly doped cuprates
}

PLEASE CITE THE PUBLISHED VERSION

PUBLISHER

(C) American Physical Society

\section{VERSION}

VoR (Version of Record)

LICENCE

CC BY-NC-ND 4.0

\section{REPOSITORY RECORD}

Alexandrov, A.S., and Kimberley Reynolds. 2019. "Angle-resolved Photoemission Spectroscopy of Band Tails in Lightly Doped Cuprates". figshare. https://hdl.handle.net/2134/4244. 
This item was submitted to Loughborough's Institutional Repository (https://dspace.lboro.ac.uk/) by the author and is made available under the following Creative Commons Licence conditions.

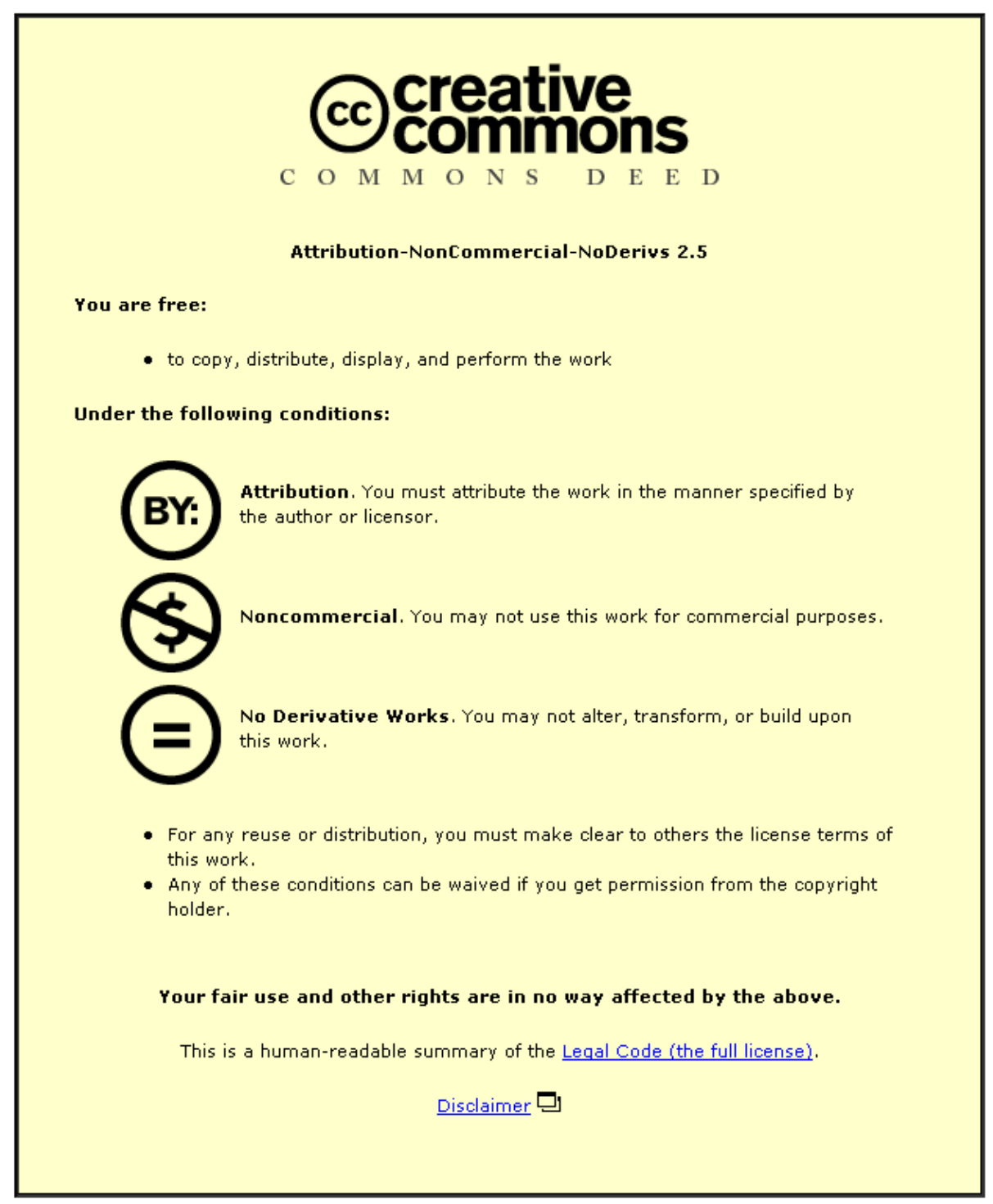

For the full text of this licence, please go to: http://creativecommons.org/licenses/by-nc-nd/2.5/ 


\title{
Angle-resolved photoemission spectroscopy of band tails in lightly doped cuprates
}

\author{
A. S. Alexandrov and K. Reynolds \\ Department of Physics, Loughborough University, Loughborough LE11 3TU, United Kingdom
}

(Received 9 August 2007; published 11 October 2007)

\begin{abstract}
We amend $a b$ initio strongly correlated band structures by taking into account the band-tailing phenomenon in doped charge-transfer Mott-Hubbard insulators. We show that the photoemission from band tails accounts for sharp "quasiparticle" peaks, rapid loss of their intensities in some directions of the Brillouin zone ("Fermi arcs"), and high-energy "waterfall" anomalies as a consequence of matrix-element effects of disorder-localized states in the charge-transfer gap of doped cuprates.
\end{abstract}

DOI: 10.1103/PhysRevB.76.132506

Since the discovery of high- $T_{c}$ superconductivity in cuprates, angle-resolved photoemission spectroscopy (ARPES) has offered a tremendous advance into the understanding of their electronic structure. ${ }^{1}$ However, even though ARPES is continually strengthening our insights into the band structure and correlations in cuprates, it has also revealed many poorly understood phenomena, such as the incoherent "background," the sharp "quasiparticle" peaks near some points of the Brillouin zone, which form "arcs" of "Fermi surface" (Ref. 2 and references therein), widely studied low-energy dip-hump and kink features (for review, see Ref. 1), and the more recently discovered steep downturn of the dispersion toward higher energies (the so-called "waterfall"). ${ }^{3-8}$ These anomalies have received quite different interpretations, involving uncorrelated ${ }^{9}$ and strongly correlated ${ }^{3,10-13}$ lattice polarons, Migdal-Eliashberg-like approaches,${ }^{14}$ spinons and holons, ${ }^{4}$ spin polarons, ${ }^{6}$ spin fluctuations,,${ }^{15,16}$ and bandstructure matrix-element effects. ${ }^{8,17}$

ARPES of undoped and lightly doped cuprates ${ }^{1-3,18-20}$ proved to be particularly critical in the assessment of different theoretical approaches. It revealed an apparent contradiction with the $t-J$ model. There is no sharp peak predicted by the model in undoped cuprates, but a slightly dispersive broad incoherent background, Fig. 1(a). Small lattice polarons due to a strong electron-phonon interaction (EPI) have been advocated as a plausible explanation of the discrepancy. ${ }^{11}$ When EPI is strong, the coherent spectral weight $Z$ of small polaron is very small, $Z \ll 1$, and, hence, the peak cannot be seen in experiment since all weight of the sharp resonance in the $t-J$ model is transformed at strong EPI into the broad continuum. However, the energy distribution curves (EDCs) in $\mathrm{La}_{2} \mathrm{CuO}_{4}$, Fig. 1(a), have only little, if any, resemblance to the small-polaron spectral function, which is roughly Gaussian-like. Only by subtracting a background given by the spectrum near $(\pi / a, \pi / a)$, Fig. 1(a), one can account for the remaining EDC with the polaronic spectral function. ${ }^{13}$ This background problem obscures a reliable interpretation of the broad ARPES intensities, especially in underdoped cuprates, where the charge-transfer gap at $2 \mathrm{eV}$ makes inelastic scattering events implausible as an explanation of the background. Sharp peaks at $(\pi / 2 a, \pi / 2 a)$ near the Fermi level, Fig. 2(b), in doped cuprates also remain a puzzle. Small heavy polarons cannot screen EPI in lightly doped cuprates. Hence, if $Z$ is small in the parent cuprate, it remains small at finite doping, so that the emergency of the peaks cannot be explained by a substantial increase of $Z$ with doping.
PACS number(s): 74.40.+k, 71.38.-k, 72.15.Jf, 74.72.-h

Here, we present a plausible alternative interpretation of ARPES puzzles in underdoped cuprates amending the strongly correlated energy band structure by in-gap band tails, inevitable in doped charge-transfer Mott insulators due to disorder and surface states.

We employ the local-density approximation plus generalized tight-binding (LDA+GTB) band structure of undoped cuprates with $a b$ initio sets of tight-binding parameters ${ }^{21}$ describing remarkably well the optical gap, $E_{c t} \approx 2 \mathrm{eV}$, both in antiferromagnetic and paramagnetic states of the undoped $\mathrm{La}_{2} \mathrm{CuO}_{4}$. The valence band consists of a set of very narrow $(\Sigma 1 \mathrm{eV})$ subbands where the highest one is dominated by the oxygen $p$ states with the maximum at $\mathbf{k} \equiv \mathbf{g}$ $=(\pi / 2 a, \pi / 2 a)$ (see Fig. 2), while the bottom of the empty conduction band formed by $d_{x^{2}-y^{2}}$ states of copper is found at $(\pi / a, 0)$. These locations of the valence-band maximum and conduction-band minimum perfectly agree with ARPES intensity locus in hole-doped $\mathrm{La}_{2-x} \mathrm{Sr}_{x} \mathrm{CuO}_{4}$ and electrondoped $\mathrm{Nd}_{2-x} \mathrm{Ce}_{x} \mathrm{CuO}_{4}$, respectively. ${ }^{18}$ Importantly, the LDA + GTB approach predicts the charge-transfer gap at any doping with the chemical potential pinned near the top of the

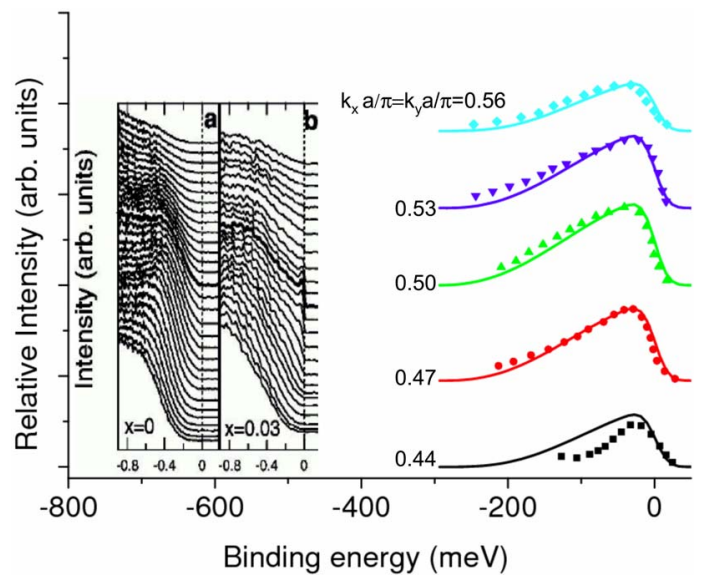

FIG. 1. (Color online) Band-tail EDC, Eq. (4), (solid lines) with pseudogap $\Delta=300 \mathrm{meV}$ and band-tail width $\gamma=300 \mathrm{meV}$ compared with relative EDC (symbols) near $(\pi / 2 a, \pi / 2 a)$. Relative intensities are obtained by subtracting ARPES intensities of the parent compound, $\mathrm{La}_{2} \mathrm{CuO}_{4}$ (a), shifted by $\delta \mu$, from EDC of slightly doped $\mathrm{La}_{1.97} \mathrm{Sr}_{0.03} \mathrm{CuO}_{4}$ (b) as measured by Yoshida et al. (Ref. 2). Both intensities have been normalized by their values at $E=$ $-800 \mathrm{meV}$, and the chemical potential shift between two samples has been taken as $\delta \mu=70 \mathrm{meV}$. 


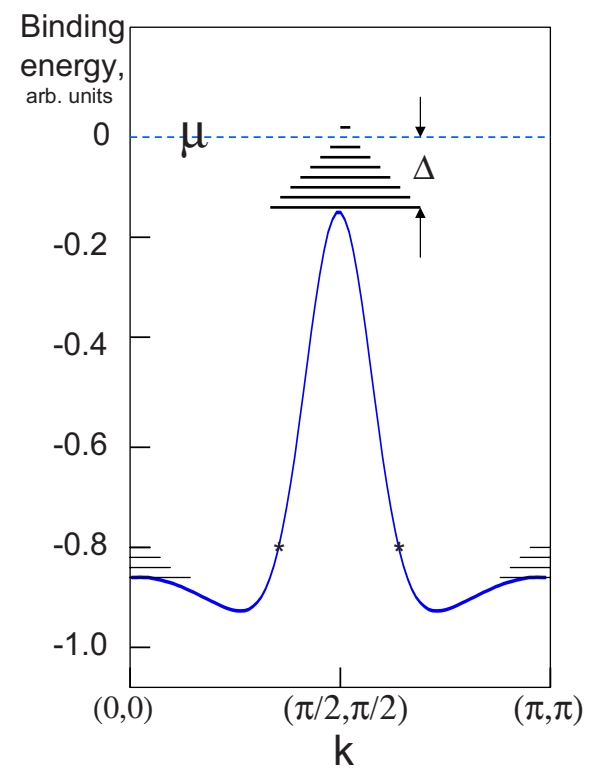

FIG. 2. (Color online) LDA+GTB valence-band dispersion (Ref. 21) amended with band tails (ladder lines) near $\Gamma,(\pi / 2, \pi / 2)$, and $(\pi, \pi)$ maxima (here, $k$ is measured in $1 / a$ ).

valence band (in hole-doped cuprates) and near the bottom of the conduction band (in electron-doped cuprates).

Doping of cuprates inserts a large number of impurities into the parent lattice. Each impurity ion locally introduces a distinct level $E_{i}$ in the charge-transfer gap. The fact that the impurities are randomly distributed in space causes the density of states (DOS) to tail, like in heavily doped semiconductors. ${ }^{22}$ When there are many impurities within the range $\xi_{i}$ of a localized wave function $\psi_{i}(\mathbf{r})$, the random potential produces low-energy states near the maxima of the valence band at hole doping, Fig. 2, or near the minima of the conduction band at electron doping. As a result, ARPES intensity, $I(\mathbf{k}, E)=I_{b}(\mathbf{k}, E)+I_{i m}(\mathbf{k}, E)$, comprises the band-tail intensity $I_{i m}(\mathbf{k}, E)$ due to localized states within the chargetransfer gap and the valence-band contribution $I_{b}(\mathbf{k}, E)$ of itinerant states. According to local-density approximation band structures, ${ }^{17}$ the itinerant states are anisotropic threedimensional (specifically in $\mathrm{La}_{2} \mathrm{CuO}_{4}$ ) dispersing with $c$-axis $k_{z}$ over a few hundred meV. We suggest that this dispersion shapes the background, making it so different from the incoherent background caused by EPI and/or spin fluctuations since $k_{z}$ is not conserved in ARPES experiments. On the other hand, the incoherent background can be well described by a simple polaronic Gaussian in presumably more anisotropic insulating $\mathrm{Ca}_{2} \mathrm{CuO}_{2} \mathrm{Cl}_{2}{ }^{20}$

Here, we focus on the band-tailing contribution described by the Fermi-Dirac golden rule as

$$
I_{i m}(\mathbf{k}, E)=\frac{2 \pi e^{2}}{m_{e}^{2}} n(E) \sum_{i}\left|\left\langle\psi_{f}\left|\mathbf{A}_{0} \cdot \nabla\right| \psi_{i}\right\rangle\right|^{2} \delta\left(E+\Delta-E_{i}\right) .
$$

We define all energies relative to the chemical potential $\mu$, which is situated at energy $\Delta$ above the top of the valence band within the impurity band as shown in Fig. 2. Only the impurity states with the binding energy $E_{i}$ below $\mu=0$ contribute at zero temperature. Here, $\mathbf{A}_{0}$ is the amplitude of x-ray vector potential, $n(E)=1 /[\exp (E / T)+1]$ is the FermiDirac distribution function, and $\hbar=k_{B}=1$.

While the LDA+GTB band dispersions fully account for the short-range Coulomb correlations in cuprates, the size of shallow impurity states is much larger than the lattice constant (as shown below in Fig. 4), so that their envelope function is roughly insensitive to the short-range correlations. Hence, we can take the impurity wave function $\operatorname{as}^{23} \psi_{i}(\mathbf{r})$ $=F_{i}(\mathbf{r}) \psi_{\mathbf{g}}(\mathbf{r})$, and the final state to be the normalized plane wave $\psi_{f}(\mathbf{r})=(N v)^{-1 / 2} \exp (i \mathbf{k} \cdot r)$. Here, $\psi_{\mathbf{g}}(\mathbf{r})$ is the itinerant state at the top of the valence band, renormalized by the correlations, and $F_{i}(\mathbf{r})$ is a slowly varying envelope function ( $N$ is the number of unit cells of volume $v$ in the crystal).

In the framework of generalized tight binding,,$^{21}$ one can expand $\psi_{\mathbf{g}}(\mathbf{r})$ using the Wannier orbitals, $\psi_{\mathbf{g}}(\mathbf{r})$ $=N^{-1 / 2} \sum_{\mathbf{m}} w(\mathbf{r}-m) \exp (i \mathbf{g} \cdot m)$, and calculate the dipole matrix element in Eq. (1) as

$$
I_{i m}(\mathbf{k}, E)=\operatorname{In}(E) \sum_{i}\left|f_{i}(\mathbf{k}-\mathbf{g})\right|^{2} \delta\left(E+\Delta-E_{i}\right),
$$

where $I=2 \pi\left(e d / m_{e}\right)^{2}\left(\mathbf{A}_{0} \cdot \mathbf{k}\right)^{2} / v$ is proportional to the valence-band matrix element squared, which is roughly a constant in a wide range of $\mathbf{k}$ near $\mathbf{g}, d=\int d \mathbf{r} w(\mathbf{r}) \exp (i \mathbf{g} \cdot r)$, and $f_{i}(\mathbf{q})=(N v)^{-1} \int d \mathbf{r} \exp (i \mathbf{q} \cdot r) F_{i}(\mathbf{r})$ is the Fourier transform of the impurity envelope function.

Since the size of the envelope is large compared with the lattice constant, its Fourier transform strongly depends on $\mathbf{q}$, which explains the experimental EDC and MDC as we show in the rest of this Brief Report. We choose the impurity state to be hydrogenlike, $F_{i}(\mathbf{r})=\left(N v / \pi \xi_{i}^{3}\right)^{1 / 2} \exp \left(-r / \xi_{i}\right)$, as the hydrogen model accurately predicts many properties of shallow levels in heavily doped semiconductors, so that $f_{i}(\mathbf{q})$ $=8 \pi\left(\xi_{i}^{3} / \pi N v\right)^{1 / 2}\left(1+q^{2} \xi_{i}^{2}\right)^{-2}$ for 3D impurity states and $f_{i}(\mathbf{q}) \propto\left(1+q^{2} \xi_{i}^{2}\right)^{-3 / 2}$ for two-dimensional (2D) states such as localized surface states. It is important to recognize here that $\xi_{i}$ is related to the impurity binding energy as $\bar{\xi}_{i}^{2}=m E_{i}$, where $m$ is roughly the hole effective mass. As a result, we get $I_{i m}(\mathbf{k}, E)=x \operatorname{In}(E) M(\mathbf{k}-g, E)$ with

$$
M(\mathbf{k}-\mathbf{g}, E)=\frac{64 \pi}{v m^{3 / 2}} \frac{(E+\Delta)^{5 / 2}}{\left[E+\Delta+(\mathbf{k}-\mathbf{g})^{2} / m\right]^{4}} \rho_{i m}(E+\Delta) .
$$

Here, $\rho_{i m}(E)=N_{i}^{-1} \Sigma_{i} \delta\left(E-E_{i}\right)$ is the band-tail density of states (DOS) normalized to unity, and $x=N_{i} / N$ is the impurity concentration per cell proportional to doping. In the $2 \mathrm{D}$ case, the result is similar, $M_{2 \mathrm{D}}(\mathbf{k}, E) \propto(E+\Delta)^{2}\left[E+\Delta+\left(\mathbf{k}_{\|}\right.\right.$ $\left.-g)^{2} / m\right]^{-3} \rho_{i m}(E+\Delta)$.

We notice that due to a very sharp dependence on $q$ of the matrix element in Eq. (2), any uncertainty of $k_{z}$ does not smear out the strong dependence of $I_{i m}(\mathbf{k}, E)$ on the in-plane momentum component $\mathbf{k}_{\|}$. Averaging over $k_{z}$ simply replaces $M(\mathbf{k}-g, E)$ in Eq. (3) by 


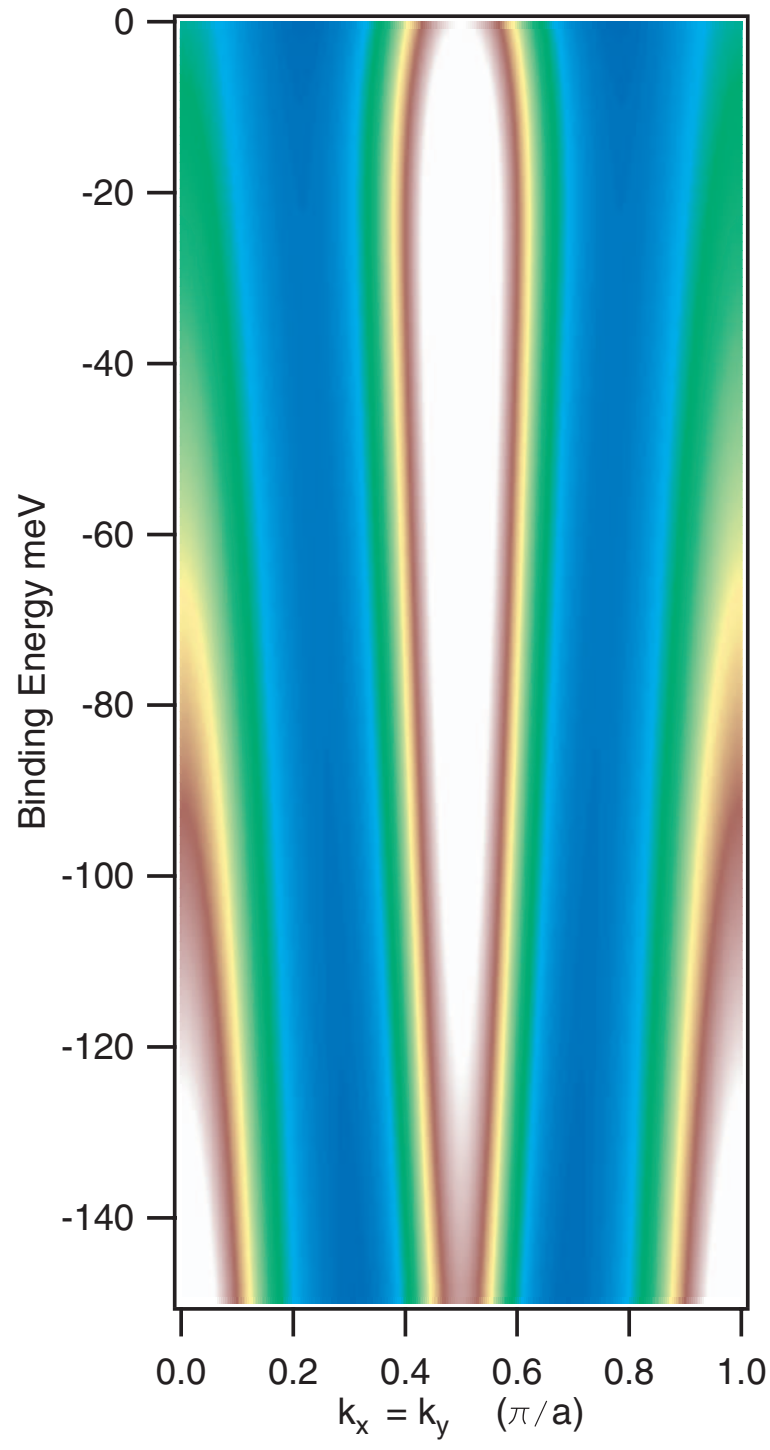

FIG. 3. (Color) Waterfall effect in the band-tail ARPES intensity (white color corresponds to the highest intensity).

$$
\tilde{M}\left(\mathbf{k}_{\|}-\mathbf{g}, E\right) \approx \frac{32 c}{v m} \frac{(E+\Delta)^{5 / 2}}{\left[E+\Delta+\left(\mathbf{k}_{\|}-\mathbf{g}\right)^{2} / m\right]^{7 / 2}} \rho_{i m}(E+\Delta),
$$

where $c$ is the $c$-axis lattice constant. Also, $M$ and $\tilde{M}$ can be very large for shallow impurity states, so that even the strong polaronic reduction of their weight, $Z \ll 1$, does not make band tails invisible in ARPES at finite doping.

Since the chemical potential shifts toward the band edge with doping, $\Delta$ in Eqs. (3) and (4) becomes smaller. Hence, the band-tail peak $I_{i m}(\mathbf{k}, E)$, which is proportional to $x$, not only increases but also becomes sharper with doping as observed. ${ }^{2}$ To provide more insight into the shape and momentum dependence of experimental EDC, we approximate the band-tail DOS by the simple form $\rho_{i m}(E)=[n / \Gamma(p / n$ $+1 / n)](E / \gamma)^{p} \exp \left(-E^{n} / \gamma^{n}\right)$, where $\Gamma(x)$ is the gamma function. Exponents $n$ and $p$ depend on the dimensionality and the correlation length of the disorder potential: $n=2$ both in
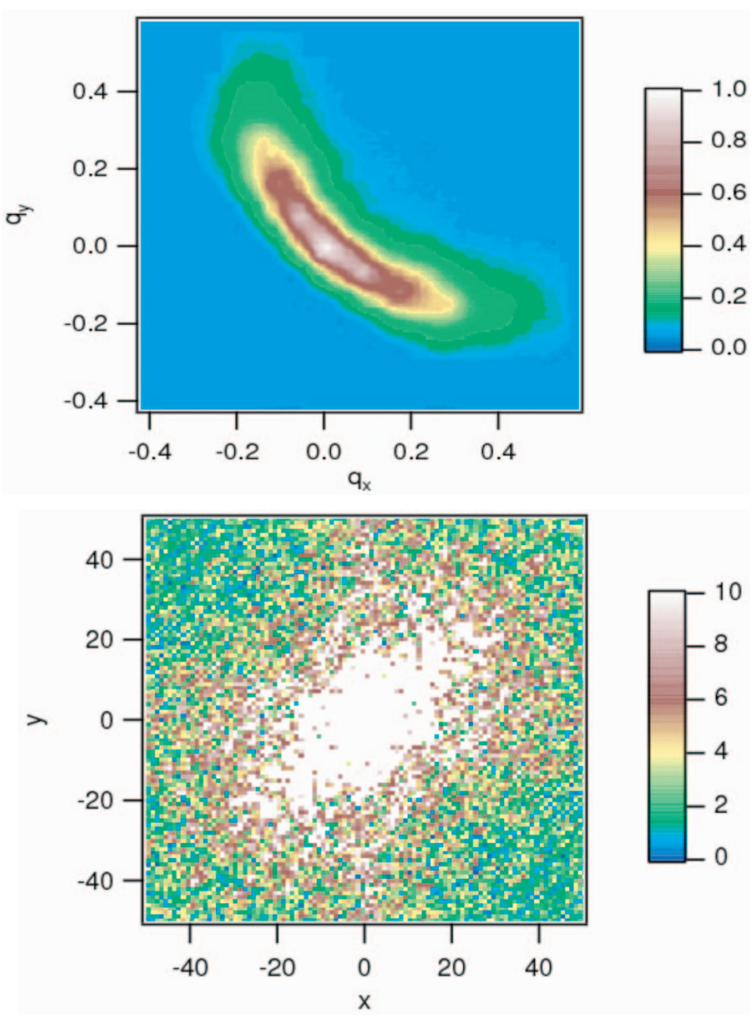

FIG. 4. (Color) Real-space Fourier transform (lower panel) of the square root of ARPES intensities [arbitrary units] at the Fermi level in $\mathrm{Ca}_{2-x} \mathrm{Na}_{x} \mathrm{CuO}_{2} \mathrm{Cl}_{2}$ [upper panel, measured by Shen et al. (Ref. 19) for $x=0.12$ ] reveals the real-space size (in units of $a$ ) of localized in-gap states.

$2 \mathrm{D}$ and $3 \mathrm{D}, p=2$ in $2 \mathrm{D}$, and $p=7 / 2$ in $3 \mathrm{D}$ for the long-range random potential correlations. In the short-range Gaussianwhite-noise limit, one obtains $n=1,1 / 2$ in $2 \mathrm{D}$ and $3 \mathrm{D}$, respectively, and $p=3 / 2$ in both dimensions. ${ }^{24}$ We can separate impurity and band contributions by subtracting normalized ARPES intensity of the parent cuprate from the intensity of the doped one. Then, the band-tail ARPES, Eq. (4), fits reasonably well the experimental relative intensities at all momenta around $\mathbf{g}$ with $m=m_{e}, n=2$, and $p=7 / 2$, Fig. 1. It describes the substantial loss of intensity with changing the momentum by only a few percent relative to $\mathbf{g}$, as well as the shape of the relative EDC.

We argue that band tailing can also contribute to the waterfall effect. There are impurity tails near local maxima of the LDA+GTB valence band at $\Gamma$ point $(0,0)$ and at $\mathbf{g}_{1}$ $=(\pi / a, \pi / a)$, as shown in Fig. 2. Different from in-gap impurity states at $\mathbf{g}=(\pi / 2 a, \pi / 2 a)$, these localized states are hybridized with the valence-band states of the same energy (shown by stars in Fig. 2). However, the hybridization could be insignificant if the corresponding matrix elements of the random potential are small due to a large momentum separation between those states of the order of $\pi / 2 a$. Hence, the impurity peaks reappear and disperse toward $(0,0)$ and $\mathbf{g}_{1}$ at high binding energies, as observed in a number of doped cuprates. ${ }^{4-8}$ We illustrate the waterfall in Fig. 3 by adding all three tail contributions, $I_{i m}(\mathbf{k}, E) \propto n(E)\left[\tilde{M}\left(\mathbf{k}_{\|}, E+E_{2}\right)+\tilde{M}\left(\mathbf{k}_{\|}\right.\right.$ $\left.-\mathbf{g}, E)+\tilde{M}\left(\mathbf{k}_{\|}-\mathbf{g}_{1}, E+E_{2}\right)\right]$, where $E_{2}$ is roughly the valence 
bandwidth (we chose $E_{2}=500 \mathrm{meV}$ ). We notice that $n(E)$ is replaced by its convolution with the Gaussian energy resolution function, $n(E) \rightarrow[1-\operatorname{erf}(E / \delta)] / 2$, in plotting Figs. 1 and 3 since the energy resolution $\delta=20 \mathrm{meV}$ is much larger than the temperature, $T \approx 2 \mathrm{meV}$. Also, the photoemission intensity comprises both band-tail and valence-band contributions, so that the resulting dispersion could be different from the anomalous band-tail dispersion of relative intensities, Fig. 1.

Our theory proposes that the ARPES intensity near $(\pi / 2 a, \pi / 2 a)$ is proportional to the square of the Fourier component $f_{i}(\mathbf{q})$ of the impurity wave-function envelope, Eq. (2). Therefore, we can find the real-space image of the function $F_{i}(\mathbf{r})$ by taking the Fourier transform of the square root of the experimental intensities, Fig. 4 (upper panel). Here, we show the intensities near the Fermi level measured in $\mathrm{Ca}_{2-x} \mathrm{Na}_{x} \mathrm{Cu} \mathrm{O}_{2} \mathrm{Cl}_{2},{ }^{19}$ which are very similar, if not identical, to those in $\mathrm{La}_{2-x} \mathrm{Sr}_{x} \mathrm{CuO}_{4}$ (compare Fig. 1 in Ref. 19 and Fig. 2 in Ref. 2). The real-space image (lower panel, Fig. 4) reveals some band-mass anisotropy, and the size of the localized state of about 20 lattice constants justifies the "envelope" approximation used for the impurity wave function. Clearly, any different shape of the envelope function considering the realistic impurity potentials could not change our conclusions as soon as its size remains large compared with the lattice constant.

In summary, we have proposed an explanation for the sharp quasiparticle peaks, Fermi arcs, and the high-energy waterfall in cuprates as a consequence of photoionization matrix element of disorder-localized band tails in the chargetransfer gap of doped Mott insulators. If holes are bound into bipolarons, the chemical potential remains within the in-gap band tails at the bipolaron mobility edge even at final doping, in agreement with $\mathrm{S}-\mathrm{N}-\mathrm{S}$ tunneling experiments. ${ }^{25}$ In this case, $\Delta$ in Fig. 2 is half of the bipolaron binding energy, which is also the normal state pseudogap. ${ }^{26}$ Remarkably, placing the chemical potential within the impurity band explains the insulatinglike low-temperature dependence of the normal state resistivity ${ }^{27}$ as well as many other kinetic and thermodynamic properties of underdoped cuprates. ${ }^{26}$ Recent scanning tunneling microscopy at the atomic scale found intense nanoscale disorder in high- $T_{c}$ superconductor $\mathrm{Bi}_{2} \mathrm{Sr}_{2} \mathrm{CaCu}_{2} \mathrm{O}_{8+\delta},{ }^{28}$ supporting the important role of disorder band tailing in shaping single-particle spectral functions of doped Mott insulators.

We are grateful to Z. X. Shen and Teppei Yoshida for providing us with their raw ARPES data (Ref. 2) and enlightening comments. We greatly appreciate valuable discussions with Arun Bansil, Sergey Borisenko, Ivan Bozovic, Jim Hague, Jan Jung, Alexander Kordyuk, Maxim Korshunov, Kyle Shen, and Jan Zaanen. This work was supported by EPSRC (UK) (Grant No. EP/C518365/1).
${ }^{1}$ A. Damascelli, Z. Hussain, and Zhi-Xun Shen, Rev. Mod. Phys. 75, 473 (2003).

${ }^{2}$ T. Yoshida et al., Phys. Rev. Lett. 91, 027001 (2003).

${ }^{3}$ F. Ronning et al., Phys. Rev. B 71, 094518 (2005).

${ }^{4}$ J. Graf et al., Phys. Rev. Lett. 98, 067004 (2007).

${ }^{5}$ W. Meevasana et al., Phys. Rev. B 75, 174506 (2007).

${ }^{6}$ B. P. Xie et al., Phys. Rev. Lett. 98, 147001 (2007).

${ }^{7}$ J. Chang et al., Phys. Rev. B 75, 224508 (2007).

${ }^{8}$ A. A. Kordyuk et al., arXiv:cond-mat/0702374 (unpublished).

${ }^{9}$ A. S. Alexandrov and C. J. Dent, Phys. Rev. B 60, 15414 (1999); A. S. Alexandrov and C. Sricheewin, Europhys. Lett. 58, 576 (2002).

${ }^{10}$ G. Wellein, H. Roder, and H. Fehske, Phys. Rev. B 53, 9666 (1996).

${ }^{11}$ A. S. Mishchenko and N. Nagaosa, Phys. Rev. Lett. 93, 036402 (2004).

${ }^{12}$ M. Hohenadler, D. Neuber, W. von der Linden, G. Wellein, J. Loos, and H. Fehske, Phys. Rev. B 71, 245111 (2005).

${ }^{13}$ O. Rosch, O. Gunnarsson, X. J. Zhou, T. Yoshida, T. Sasagawa, A. Fujimori, Z. Hussain, Z.-X. Shen, and S. Uchida, Phys. Rev. Lett. 95, 227002 (2005).

${ }^{14}$ J. P. Hague, J. Phys.: Condens. Matter 15, 2535 (2003).

${ }^{15}$ S. V. Borisenko et al., Phys. Rev. Lett. 96, 117004 (2006).

${ }^{16}$ A. Macridin, M. Jarrell, T. Maier, and D. J. Scalapino, arXiv:cond-mat/0701429 (unpublished).
${ }^{17}$ M. Lindroos, S. Sahrakorpi, and A. Bansil, Phys. Rev. B 65, 054514 (2002).

${ }^{18}$ N. P. Armitage et al., Phys. Rev. Lett. 88, 257001 (2002); K. M. Shen et al., Phys. Rev. B 69, 054503 (2004).

${ }^{19}$ K. M. Shen et al., Science 307, 901 (2005).

${ }^{20}$ K. M. Shen et al., Phys. Rev. B 75, 075115 (2007).

${ }^{21}$ S. G. Ovchinnikov, A. A. Borisov, V. A. Gavrichkov, and M. M. Korshunov, J. Phys.: Condens. Matter 16, L93 (2004); M. M. Korshunov, V. A. Gavrichkov, S. G. Ovchinnikov, I. A. Nekrasov, Z. V. Pchelkina, and V. I. Anisimov, Phys. Rev. B 72, 165104 (2005).

${ }^{22}$ P. V. Mieghem, Rev. Mod. Phys. 64, 755 (1992).

${ }^{23}$ W. Kohn and J. M. Luttinger, Phys. Rev. 97, 869 (1955).

${ }^{24}$ B. I. Halperin and M. Lax, Phys. Rev. 148, 722 (1966); R. Eymard and G. Duraffourg, J. Phys. D 6, 66 (1973); D. N. Quang and N. H. Tung, Phys. Status Solidi B 209, 375 (1998).

${ }^{25}$ I. Bozovic, G. Logvenov, M. A. J. Verhoeven, P. Caputo, E. Goldobin, and T. H. Geballe, Nature (London) 422, 873 (2003).

${ }^{26}$ A. S. Alexandrov, in Studies in High Temperature Superconductors, edited by A. V. Narlikar (Nova Science, New York, 2006), Vol. 50, pp. 1-69.

${ }^{27}$ A. S. Alexandrov, Phys. Lett. A 236, 132 (1997).

${ }^{28}$ J. Lee et al., Nature (London) 442, 546 (2006). 